\title{
Factores condicionantes de la deserción universitaria
}

\section{Mirna Elizabeth Miño de Gauto}

mireliza29@gmail.com

Facultad de Humanidades y Ciencias de la Educación

Universidad Nacional de Pilar

\section{RESUMEN}

La deserción universitaria es uno de los fenómenos que atenta al logro de la calidad educativa, este hecho se percibe en la sociedad actual y posee consecuencias no deseadas por el alto costo social, económico y personal. El objetivo del presente trabajo consiste en analizar bibliográficamente los principales factores de la deserción universitaria. Se justifica considerando la relevancia a nivel teórico y práctico, el mismo constituye una realidad: la deserción y la necesidad de contextualizar las condiciones que permiten esta situación. La metodología respondió a la revisión bibliográfica, recopilación de materiales referentes al tema, se realizó una búsqueda de artículos en las principales bases de datos disponibles, portal CICCO y en Google Académico. Se adoptó el enfoque cualitativo, con diseño analítico, descriptivo. El resultado del análisis demostró que existen factores internos y externos que convergen en la deserción universitaria por lo que amerita la implementación de múltiples transformaciones, tanto a nivel de las instituciones de educación superior como así también de los organismos encargados de este nivel, que aseguren políticas de desarrollo de la calidad con el abordaje de la deserción como una de las principales líneas de acción.

Palabras clave: deserción universitaria; educación superior; calidad educativa 


\title{
Conditioning factors for university dropouts
}

\begin{abstract}
University dropout is one of the phenomena that threatens the achievement of educational quality, this fact is perceived in today's society and has unwanted consequences due to the high social, economic and personal cost. The objective of this work is to analyze bibliographically the main factors of university dropout. It is justified considering the relevance at a theoretical and practical level, it constitutes a reality: desertion and the need to contextualize the conditions that allow this situation. The methodology responded to the bibliographic review, compilation of materials relate to the subject, an article search was carried out in the main available databases, CICCO portal and in Google Scholar. The qualitative approach was adopted, with an analytical, descriptive design. The result of the analysis showed that there are internal and external factors that converge in university dropout, which warrants the implementation of multiple transformations, both at the level of higher education institutions as well as the agencies in charge of this level, which ensure quality development policies with the approach to desertion as one of the main lines of action.
\end{abstract}

Keywords: university dropout; higher education; educational quality

Artículo recibido: 10. Junio. 2021 Aceptado para publicación: 16. Julio. 2021 Correspondencia: mireliza29@gmail.com Conflictos de Interés: Ninguna que declarar 


\section{INTRODUCCIÓN}

La deserción estudiantil es entendida según Smulders Chaparro, (2018) como uno de los problemas que aborda la mayoría de las instituciones de educación superior en numerosos países. A través de distintas investigaciones, se da cuenta de un número importante de estudiantes que no logran culminar sus estudios universitarios, con el consecuente costo social asociado a este fenómeno.

Esta situación presenta un grado de complejidad, a juzgar por los antecedentes teóricos analizados en el presente trabajo de investigación, en esta problemática convergen múltiples ámbitos de la vida no sólo económica, social y política de cualquier nación, sino, de manera preponderante a seres humanos que alguna vez tuvieron la aspiración de mejorar no sólo sus condiciones de vida, sino la de sus familias a través del ingreso y culminación de una carrera profesional que les permitiera obtener un título universitario $\mathrm{y}$ "ser alguien en la vida" y que, desafortunadamente, ven frustradas dichas aspiraciones y sueños al quedar fuera del sistema educativo (Medina \& Gaytan, 2020).

Así mismo, con el fin de motivar la graduación de los estudiantes en el tiempo teórico de los programas académicos, es importante que las instituciones de educación superior, en particular las públicas, emprendan campañas pedagógicas sobre la importancia de terminar los estudios en el tiempo teórico haciendo énfasis en los costos que representa tanto para los estudiantes y sus familias como para las instituciones y la sociedad el retraso en la obtención de un título profesional.

Adicionalmente, otra política orientada al favorecimiento de la finalización exitosa de los estudios podría ser la mayor promoción de los programas de semillero, los cuales unidos a una apropiada orientación profesional pueden promover tanto la graduación como la retención estudiantil.

En el presente trabajo se buscó responder a la siguiente interrogante:

¿Cuáles son los factores que influyen en la deserción universitária?

La deserción universitaria emerge como un fenómeno multifactorial alarmante porque presenta consecuencias en diferentes planos y para distintos actores: estudiantes, instituciones y el Estado.

La deserción estudiantil es uno de los fenómenos sociales y educativos que se observa en las diversas sociedades, en menor o mayor grado, ante ello, se han realizado diversos estudios que intentan responder que factores influyen para que el estudiante dejen de asistir o formar parte de una institución educativa. (Medina \& Gaytan, 2020) 


\section{Apaza y Huamán (2012) indicaron que:}

La deserción de estudiantes se puede dar por diversos motivos, se focaliza en estudiantes menos motivados y con pocas expectativas respecto de las recompensas derivadas de la graduación, son ellos los que tienen más probabilidades de abandonar el centro de estudios. Además, las instituciones de baja calidad, según lo perciben los estudiantes, tienden a presentar tasas de deserción más altas. Otros aspectos importantes incluyen el currículo, el tamaño, la proporción de alumnos por maestro, la infraestructura y un exceso de contenido (como se citó en (Ponce de León, 2018) p.79)

La deserción estudiantil es considerada como el abandono definitivo de la formación académica por parte de los estudiantes, independientemente de las condiciones y la modalidad (Paramo, 1999 como se citó en Camacho,et. al, 2019) Este fenómeno es considerado un problema para el Sistema Educativo, lo que hace obligatorio que en las instituciones se establezcan mecanismos de ajuste a la vida universitaria de sus estudiantes, impactando así en el éxito académico. Por tal razón, es importante desarrollar estudios que permitan determinar la dimensión del problema en la institución educativa y de acuerdo a ello poder establecer con la debida anticipación, estrategias orientadas a lograr la finalización del programa que se está cursando. La graduación estudiantil es el reflejo del cumplimiento de los objetivos fundamentales de la Educación Superior y se logra a partir del desarrollo de procesos formativos y la implementación de estrategias de intervención pertinentes en cada etapa del ciclo de formación del estudiante (Ministerio de Educación Nacional, 2015 como se citó en Camacho, et. al, 2019.

\section{Factores asociados a la desercion}

Apaza y Huamán (2012) describieron los principales factores asociados a la deserción de estudiantes de nivel superior, destacando los siguientes:

Factor psicológico: Los procesos psicológicos son individuales, aunque rasgos como la incapacidad de adaptarse a un nuevo ambiente es un indicador de que el estudiante no podrá enfrentar el reto que supone estar en una institución de enseñanza superior, generando en muchas ocasiones la deserción estudiantil (Apaza y Huamán, 2012, p.82). (como se citó en Ponce de León, 2018) 
Factor social: El factor social está vinculado también al aspecto económico, siendo una valla para muchos estudiantes de seguir estudiando, ya que al formar parte de un curso o carrera no solo implica tiempo, sino recursos que deben ser afrontados por el estudiante o su entorno. Dentro del aspecto social resalta a su vez, las capacidades que posee cada estudiante, no habiendo homogeneidad, ya que en la actualidad la educación se está volviendo tecnológica, con base en la informática, y si el estudiante no cuenta con los conocimientos básicos se les dificulta estar en el mismo nivel de comprensión y aprendizaje que sus compañeros, siendo un determinante de deserción (Apaza y Huamán, 2012, p.83como se citó en Ponce de León, 2018)

Factor institucional: Este factor sostiene que la deserción se vincula con las cualidades de la institución, evidenciado en la infraestructura, espacios, ambientes de estudio, y sobre todo la calidad de la docencia. Cuando una institución no provee al alumno de elementos básicos para que pueda desarrollarse y desenvolverse, se genera rechazo a la institución, habiendo caso de deserción por este factor teorías relacionadas a la deserción de estudiantes (Apaza y Huamán, 2012, p.83, como se citó Ponce de León, 2018).

\section{Perspectivas asociadas a la deserción}

\section{Enfoque psicológico}

La característica principal se relaciona con los rasgos de personalidad que diferencian a los estudiantes que completan sus estudios de aquellos que no.

Teoría de la Acción Razonada: refiere que el comportamiento está significativamente influenciado por creencias y actitudes. Por lo tanto, la decisión de salir o continuar en un programa académico es influenciado por: el comportamiento en el nivel secundario, actitudes sobre el abandono y persistencia, y reglas subjetivas sobre estas acciones, "generando una línea de comportamiento de ambigüedad, dejadez e indiferencia que se adopta al enfrentar situaciones de la vida" (Viale, 2014, p.21), describe que los comportamientos de las personas son directamente influenciados por su sistema de creencias, lo que marca la diferencia entre los desertores estudiantiles y los que completan sus estudios universitarios.

Debido a esto, rasgos de la personalidad como la dejadez y la indiferencia pueden influir negativamente en la decisión de abandonar o continuar los estudios universitarios. (Viale, 2014) 


\section{Enfoque sociológico}

Los primeros modelos en abordar la deserción estudiantil se dieron desde una perspectiva sociológica. Estas enfatizan la influencia de factores externos al individuo en abandonar el centro de estudio, los que se suman a lo psicológico.

Entre las teorías que sustentan este enfoque se destaca la propuesta de Spady (1971) sobre la deserción académica, quien argumenta que el abandono tiene que ver con las pocas oportunidades que tiene el estudiante, y que es absorbido por sus problemas en este sentido destaca que junto a ello se analiza el entorno, la deserción aumenta con la incapacidad para integrarse en la sociedad es así que la probabilidad de deserción aumenta cuando hay una conciencia moral baja (baja coherencia regulatoria) e insuficiente afiliación social. (como se cito en Viale, 2014)

Viale (2014) destaca que el bajo apoyo en las relaciones sociales influye para el aumento de la deserción, en este sentido sostiene que "la incapacidad para integrarse en la sociedad, la baja conciencia moral y el apoyo escaso de las relaciones sociales son aspectos que promueven las deserciones estudiantiles" (p.61). Adicionalmente, los factores externos como las pocas oportunidades y los problemas que puede tener el estudiante, son motivos para considerar el abandono de los estudios.

\section{Enfoque económico}

El enfoque económico responde a la aplicación del enfoque de costo-beneficio se afirma que la racionalidad de los beneficios no siempre funciona como se esperaba, es decir, cuando los beneficios sociales y económicos generados por la universidad se percibe como inferior a los derivados de actividades alternativas, las personas eligen retirarse; es decir se declinan en seguir trabajando, ya que al percibir un sueldo y manejar dinero se dejan absorber por este sistema (Viale, 2014, p.61)

Una de las teorías que fundamenta este enfoque es argumentado por Román (2013), quien sostiene que: Debemos mirar la deserción como un fenómeno doloroso en la vida de las personas, que afecta la construcción del proyecto de vida, e incide en la propia autovaloración del sujeto. La decisión de abandonar definitivamente los estudios, es un proceso fuertemente asociado al universo de significaciones de los jóvenes, construido y validado en el transcurso de su vida cotidiana y su trayectoria académica (p. 54).

Tinto (1987), argumenta que la deserción no es una condición individual "es un reflejo del contexto social y académico" (p. 42) y es aquí donde entran en juego las visiones y 
acciones de las instituciones, encaminadas a tomar en cuenta las condiciones estructurales de los sistemas económicos, políticos y socioculturales para brindar apoyo y acompañamiento a los sectores estudiantiles más vulnerables que logran ingresar a las aulas universitaria

Esta situación atribuye el autor Vargas (2015) a que "los estudiantes que culminaron su ciclo de bachillerato aún no tienen una idea clara de lo que pretenden hacer en la universidad" (p. 19).

La falta de vinculación con el nivel educativo que le antecede a los estudios universitarios, aunado a la falta de información a los estudiantes por parte de las universidades antes de ingresar formalmente a los programas de estudios, en muchas ocasiones pueden tener como consecuencia la elección incorrecta de la carrera y, esto parece ser un factor muy importante para que los estudiantes de nivel superior no puedan concluir satisfactoriamente su formación profesional, conllevando en muchas ocasiones a la deserción (Vargas ,2015).

Los estudiantes que no optaron por una carrera correcta y a su vez tienen un concepto erróneo de la misma, son los que, en repetidas ocasiones, tienen que abandonar la institución (González, 2016; Hernández, Mogollón, Ramírez y Sierra, 2017, como se citó en (Medina \& Gaytan, 2020)

En consecuencia, las expectativas que tienen los estudiantes acerca de la universidad están fuera de la realidad, produciendo un fracaso inminente que termina en deserción. Hay una diferencia muy marcada entre las elevadas expectativas cuando los estudiantes ingresan a la universidad, pero, una vez dentro, no pueden alcanzar las exigencias de la institución y provocan el abandono de los estudios por falta de integración académica y metas institucionales, además de ocasionar malestar, incomunicación, ausentismo y apatía (Febles, Guzmán y Tuyub, 2014; Buentello, 2013; Barragán, González y Calderón, 2018, como se citó en (Medina \& Gaytan, 2020)

En muchas ocasiones la práctica docente es considerada por el alumnado como rutinaria y aburrida, además de ser muy tradicional y ancestral (Buentello, 2013; González, 2016). Los docentes no se actualizan, provocando en los estudiantes poco interés en los modelos educativos, además, na van acorde a los cambios propios de los contextos actuales. Por ejemplo, todavía hay docentes que no manejan las herramientas tecnológicas en clases, haciéndolas tediosas y aburridas (Buentello, 2013; González, 2016). En el mismo sentido, 
se han identificado ciertos factores que tienen un predominio en la deserción, entre ellos, los estilos de ser docente, los diseños de los planes de estudio fraccionados y fuera de contexto lo que no permite la articulación y la unificación de los conocimientos (Corominas, 2001; Buentello, 2013; Pérez y López, 2016 como se citó en, Medina \&Gaytan, 2020)

\section{ESTRATEGIAS METODOLÓGICAS O MATERIALES Y MÉTODOS}

Esta revisión documental forma parte de la conformación del estado del arte en la primera fase de un estudio de tesis doctoral más amplio, y de profundo análisis del tema. Para esta investigación bibliográfica se procedió a la revisión y el análisis de la literatura referente a la deserción en el nivel universitario, la metodología de búsqueda de la información bibliográfica fue mediante la recopilación de materiales referentes al tema, se realizó una búsqueda de artículos en las principales bases de datos disponibles, portal CICCO y en Google Académico, las informaciones de los diferentes autores fueron recogidos mediante la organización de fichas bibliográficas y una planilla Excel para destacar los aspectos más importantes de las informaciones y de los autores.

Este proceso de búsqueda y organización de los diferentes materiales fue de vital importancia para conocer y estudiar a diferentes autores que abordan la presente problemática

\section{DISCUSIÓN}

A fin de responder a la pregunta que originó el presente trabajo se destaca cuanto sigue:

El abordaje de los factores que causan la deserción resultan ser diversos, tanto como los investigadores que han abordado la temática, se han realizado abordajes desde el estudiante y sus motivaciones (Poveda, 2019).

Existe la necesidad de abordar el estudio de la problemática de la deserción universitaria desde un criterio multidimensional que al mismo tiempo de incorporar las conceptualizaciones de los modelos resulte ser integrador de los mismos. (Poveda, 2019)

$\mathrm{Al}$ analizar los factores que influyen en la deserción se observaron que existen varias clases de deserción escolar, entre ellas cuando el estudiante decide abandonar de forma voluntaria sus estudios (deserción total), cuando decide cambiar de carrera o de universidad (deserción por facultad), la no adaptación a la vida universitaria (deserción durante el primer semestre de la carrera), por escoger otra carrera pero dentro de la misma 
universidad (deserción por programa) (Páramo y Correa, 2012).Como se citó en (Alcalá Castro, 2019).

Luego del análisis exhaustivo de las referencias bibliográficas, se puede organizar de la siguiente manera:

\section{Factores internos}

Los factores internos que afectan a la deserción constituyen el estado civil, calificaciones de los niveles anteriores, expectativas de la carrera no cubiertas, la falta de una orientación vocacional, el no adaptarse al ámbito universitario y las dificultades de ingreso, entre otros (Maya, 2009). (Smulders Chaparro, 2018)

El factor interno, individual y/o psicológicos también puede causar la deserción; como ser la falta de orientación vocacional, las dificultades emocionales, la falta de resistencia al estrés, la autoestima, el temperamento, el coeficiente intelectual y la perseverancia; que van asociadas a la deserción universitaria (Himmel, 2001), (Smulders Chaparro, 2018)

Viale (20149 destaca rasgos en la personalidad que diferencian a los estudiantes que completan sus estudios de aquellos que no.

\section{Factores externos}

Entre los autores que mencionan los factores externos se ha encontrado a Ponce de León (2018) quien señala al fator social e institucional como elementos que inciden en la deserción, así mismo Viale (2014), Román (2013), señalaron a los factores externos como ser los aspectos sociológicos, económico e institucionales inciden en mayor medida en la deserción universitária.

En este ámbito Alcalá Castro (2019) sitúa a la dimensión académica asociado al potencial o capital cultural y académico con el cual ingresan los estudiantes a la educación superior, como determinantes de esta difícil situación que enfrenta la Educación Superior 


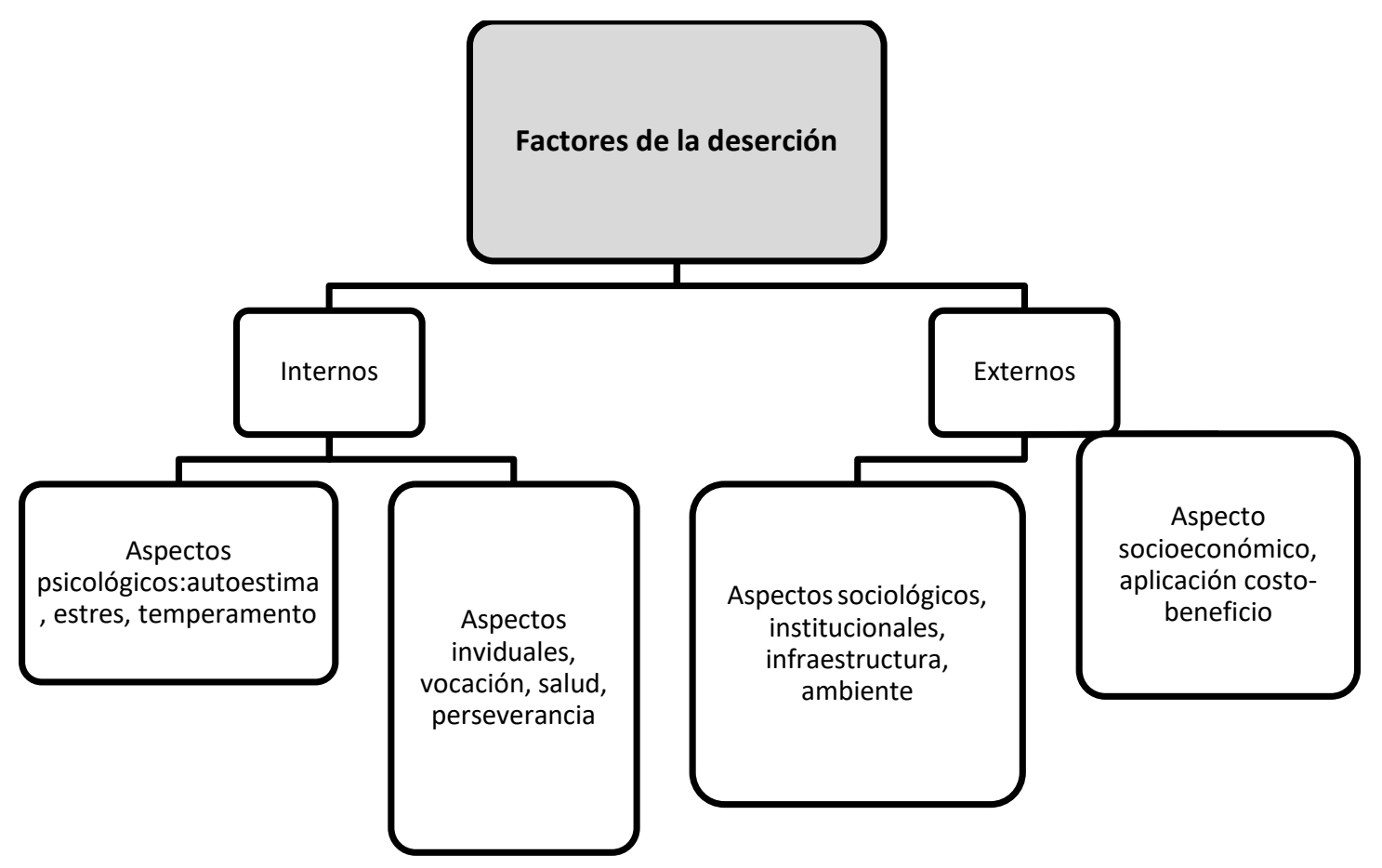

Fuente: elaboración propia

\section{Convergencia de los factores en el discente universitario}

Luego del análisis de los factores mencionados, es menester destacar lo señalado por Tinto (1975) quien plantea que la deserción es causada por la interacción de elementos individuales, sociales e institucionales. (Factores internos y externos interactúan) (Tinto, 1975)

En relación con los aspectos sociales, el autor destaca la centralidad de las características o antecedente familiar de los estudiantes, siendo especialmente significativos aquellos relacionados con la ocupación y nivel educacional de los padres, la valoración y expectativas educativas de los jóvenes y el compromiso con una meta de estudios y con futuros objetivos. (Tinto, 1975)

\section{CONCLUSIÓN O CONSIDERACIONES FINALES}

El presente trabajo aborda, los diferentes factores que inciden en la deserción de los estudiantes universitarios, este análisis presenta un grado de complejidad de singular significado en la que convergen factores internos y factores externos al estudiante, que amerita una reflexión profunda por los responsables de la Educación Superior, considerando que los mismos son seres humanos, compatriotas que aspiran una vida profesional para mejorar la calidad de vida de sí mismo y del entorno. 
Cabe destacar que entre los factores externos mencionados se encuentra la Institución, el aspecto académico organizacional, por lo tanto, amerita una revisión profunda de las autoridades de acompañar y asesorar a los estudiantes para que éstos puedan cumplir la meta de ser graduados universitarios, que no sean meras estadísticas de abandono sin conocer las causas y sin preparar estrategias que puedan paliar estas situaciones

Con relación al factor interno, también las Instituciones de educación Superior pueden proponer diversos talleres a los estudiantes desde los primeros años en relación a la autoestima, sobre la vocación, la ansiedad, el estrés, a fin de apoyar la inserción y graduación efectiva de los estudiantes.

Es así que con el estudio teórico del tema se observa en los últimos años la consolidación de mecanismos de aseguramiento de la calidad, en este sentido destacan Lemaitre et al. (2018) que es necesario la implementación de múltiples transformaciones, tanto a nivel de las instituciones de educación superior como así también de los organismos encargados de este nivel, que aseguren políticas de desarrollo de la calidad, transitando de procedimientos rutinarios a una verdadera cultura de la calidad, donde la deserción universitaria se ubique entre las prioridades educativas que debe ser entendida desde diferentes perspectivas, en la que converge el control, la garantía institucional y por sobre todo el compromiso por la mejora permanente hacia la calidad.

\section{LISTA DE REFERENCIAS}

Agencia Nacional de Evaluación y Acreditación, d. (septiembre de 2013). Modelo Nacional de Evaluación y Acreditación, de la Educación Superior. Recuperado el 16 de Julio de 2019, de Criterios de Calidad para la Carrera de Contaduría Pública: www.ANEAES.COM

Alcalá Castro, A. (2019). Validaciónde un instrumento para la identificación de las determinantes causales de la deserción estudiantil . Universidad del bosque, 164.

Camacho, M., Montalvo, A., \& Galezo, P. (2019). Determinantes de la desercion estudiantil en estudiantes universitarios. Panorama Economico, 1-17.

Celaya Figueroa, R., López Parra, M. E., \& Aceves López, J. N. (2008). Ética docente del contador público: un análisis comparativo internacional. Cuad. Contab., 4156. 
Damián, J. (2014). Identidad profesional, reconocimiento social e inserción laboral del universitario con formación híbrida. Propósitos y Representaciones, 9 - 43.

Díaz Peralta, C. (2008). Modelo conceptual para la deserción estudiantil. Revista Estudios pedagógicos , 65-86.

Gette, M. Á., Pordomingo, E., Rodriguez, R., \& Antonietti, L. (2018). INSERCIoN LABoRAL Y TRAYECToRIA PRoFESIoNAL DE LOS CoNTADoRES PUBLICoS. PERSPECTIVAS de las Ciencias Económicas y Jurídicas, 81 - 98.

Ghenny, C. M. (2014). Factores que inciden en la deserción estudiantil en el programa académico administración de empresas. Tesis doctoral. Universidad del Valle. Sede Pacífico, 1-112.

Gómez Cano, C. A., Sánchez Castillo, V., Fajardo, M. Y., Trucco, G. G., \& Cifuentes Garzón, D. (2016). CARACTERIZACIÓN DE LOS GRADUADOS DEL PROGRAMA DE CONTADURÍA PÚBLICA DE LA UNIVERSIDAD DE LA AMAZONIA 2013-2015. FACE Facultad de Ciencias Económicas y Empresariales, 29-37.

Gómez Sánchez, D., Oviedo Marín, R., \& Martínez López, E. Í. (2011). Factores que influyen en el rendimiento académico del estudiante universitario. Educación y Humanidades, 90-97.

Hernández Sampieri, R., Fernández, C., \& Baptista, P. (2006). Metodología de la Investigación. México: Mc Graw Hill.

Jaramillo, A., Giraldo Pineda, A., \& Ortiz Correa, J. S. (2006). Estudios sobre Egresados: la Experiencia de la Universidad EAFIT. Centro de Investigaciones Económicas y Financieras, 111-124.

Lemaitre, M., Aguilera, R., Dibbern, A., Hayte, C., Muga, A., \& Téllez, J. (2018). La educación superior como parte del sistema educativo de América Latina y el Caribe. Calidad y Aseguramiento de la calidad. Conferencia Regional de educación Superior. Córdoba. Argentina: UNESCO-IESALC y UNC.

Lemaitre, M., Aguilera, R., Hayte, C., Muga, A., \& Téllez, J. (2018). La educación superior como parte del sistema educativo de América Latina y el Caribe. Conferencia Regional de Educación SUperior. UNESCO. 
Marulanda Galvis, J. C., Ortiz Botero, E., Moratto Vásquez, N., \& Arcila Rojas, A. P. (2010). Caracterización de egresados de la Universidad CES en las cohortes de 2003, 2005, 2007 y momento “0”. Revista CES Psicología, 50-63.

Medina, M., \& Gaytan, C. (2020). Aspectos que inciden en la deserción universitaria, un análisis crítico. Revista Educar, 1-18.

Molina Gutiérrez, T. D., Arciniegas P., O. G., \& Pantoja Burbano, M. J. (2018). Formación Universitaria y Oportunidades Laborales. Seguimiento a los graduados en Contaduría - Auditoría. Horizontes de la Ciencia, 161-174.

Nieves Clavijo, M. E. (2008). Factores de deserción estudiantil en el Programa de Contaduría Pública. Tesis doctoral. Universidad Libre. Colombia, 1-97.

Ponce de León, A. (2018). Factores que influyen en el nivel de deserción de los estudiantes del programa de actualización y perfeccionamiento de la Academia de la Magistratura. Universidad San Ignacio de Loyola , 1-33.

Poveda, I. (2019). Los factores que influyen sobre la deserción universitaria. Estudio en la UMRPSFXCh - Bolivia,análisis con ecuaciones estructurales. Revista de investigación y negocios, 1-17.

Ramírez Caballero, R. I., \& Brizuela Bordón, M. (2002). Caracterización sociodemográfica, perfil laboral y bienestar del profesional universitario en en Paraguay. Evidencia del Censo 2002, 40-63.

Real Academia Española. (16 de Julio de 2019). Real Academia Española. Obtenido de www.rae.es

Seminara, M. P. (2020). Ladesersion universitaria:resilencia como posibilidad de logro. Revista digital.

Smulders Chaparro, M. (2018). Factores que influyen en la deserción de los estudiantes universitarios. Revista Academo, 1-6.

Tinto, V. (1975). Dropout From Higher Education: A Theoretical Synthesis of Recent Research. Review of Educational Research , 45, 89-125.

Varón García , J. K. (2016). La competencia desleal un Problema de Ética Profesional. Universidad Militar Nueva Granada, 1-16.

Viale, H. (2014). Una aproximación teórica de la deserción estudiantil universiratia. Revista Digital Ridu, 1-18. 